\title{
Characterisation of the radio frequency spectrum emitted by high energy air showers with LOFAR
}

L. Rossetto*1 , A. Bonardi ${ }^{1}$, S. Buitink ${ }^{2}$, A. Corstanje ${ }^{1}$, H. Falcke ${ }^{1,3,4}$, B.M. Hare ${ }^{5}$, J.R. Hörandel ${ }^{1,3}$, P. Mitra ${ }^{2}$, K. Mulrey ${ }^{2}$, A. Nelles ${ }^{1,6}$, A. Olid Gonzalez ${ }^{1}$, J.P. Rachen ${ }^{1}$, P. Schellart ${ }^{1,7}$, O. Scholten ${ }^{5,8}$, S. ter Veen ${ }^{1,4}$, S. Thoudam ${ }^{1,9}$, T.N.G. Trinh $^{5}$, T. Winchen ${ }^{2}$

${ }^{1}$ Department of Astrophysics/IMAPP, Radboud University, P.O. Box 9010, 6500 GL Nijmegen, The Netherlands

${ }^{2}$ Astrophysical Institute, Vrije Universiteit Brussel, Pleinlaan 2, 1050 Brussels, Belgium

${ }^{3}$ NIKHEF, Science Park Amsterdam, 1098 XG Amsterdam, The Netherlands

${ }^{4}$ Netherlands Institute of Radio Astronomy (ASTRON), Postbus 2, 7990 AA Dwingeloo, The Netherlands

${ }^{5}$ KVI-CART, University Groningen, P.O. Box 72, 9700 AB Groningen, The Netherlands

${ }^{6}$ Department of Physics and Astronomy, University of California Irvine, Irvine, CA 92697-4575, USA

${ }^{7}$ Department of Astrophysical Sciences, Princeton University, Princeton, NJ 08544, USA

${ }^{8}$ Interuniversity Institute for High-Energy, Vrije Universiteit Brussel, Pleinlaan 2, 1050 Brussels, Belgium

${ }^{9}$ Department of Physics and Electrical Engineering, Linnéuniversitetet, 35195 Växjö, Sweden

E-mail: 1.rossettodastro.ru.nl

The high number density of radio antennas at the LOFAR core in Northern Netherlands allows to detect radio signals emitted by extensive air showers in the energy range $10^{16}-10^{18} \mathrm{eV}$, and to characterise the geometry of the observed cascade in a detailed way. The radio signal emitted by extensive air showers along their propagation in the atmosphere has been studied in the $30-70 \mathrm{MHz}$ frequency range. The study has been conducted on real data and simulated showers. Regarding real data, cosmic ray radio signals detected by LOFAR since 2011 have been analysed. For simulated showers, the CoREAS code, a plug-in of the CORSIKA particle simulation code, has been used. The results show a clear dependence of the frequency spectrum on the distance to the shower axis for both real data and simulations. In particular, the spectrum flatten at a distance around $100 \mathrm{~m}$ from the shower axis, where the coherence of the radio signal is maximum. This behaviour could also be used to reconstruct the position of the shower axis at ground. A correlation between the frequency spectrum and the geometrical distance to the depth of the shower maximum $\mathrm{X}_{\max }$ has also been investigated. The final aim of this study is to find a method to improve the inferred information of primary cosmic rays with radio antennas, in view of affirming the radio detection technique as reliable method for the study of extensive air showers.

$35^{\text {th }}$ International Cosmic Ray Conference,

12 - 20 July, 2017

Bexco, Busan, Korea

${ }^{*}$ Speaker. 


\section{Introduction}

Radio emission from Extensive Air Showers (EAS) was detected for the first time by Jelley et al. in 1965 [1]. Since 2005 radio experiments like CODALEMA [2] and LOPES [3] started detecting air showers up to an energy of $10^{18} \mathrm{eV}$, confirming the radio emission mechanisms of cosmic rays in the Earth atmosphere. Measurements performed in the last years by the LOFAR experiment [4], the Auger Engineering Radio Array of the Pierre Auger Observatory (AERA) [5], and the Tunka-Rex radio array [6] have greatly improved the understanding of radio emission processes of extensive air showers. First studies of radio frequency spectra below $100 \mathrm{MHz}$ were conducted in late 1960s and early 1970s [7]. Analytical calculations [8, 9] and simulation studies [10] conducted at the beginning of years 2000s indicated a dependence of the radio frequency spectrum on cosmic ray air shower characteristics. Furthermore, recent analysis conducted at AERA shows a dependence of the frequency spectrum on primary particle properties [11]. This demonstrates that it is feasible to extrapolate information from the frequency spectrum with the current radio antenna arrays.

A detailed study for characterising the pattern of the radio frequency spectrum and studying its correlation with the primary cosmic ray features has been conducted with LOFAR. Among radio experiments, LOFAR is the one with the densest number of antennas, thus the most suitable for this kind of analysis. In order to find the best parameters which describe the correlation between primary cosmic rays and the emitted radio signals, data collected by LOFAR since 2011 have been studied in the $30-70 \mathrm{MHz}$ frequency range. Results obtained on real data have been cross-checked with simulations.

\section{The LOw Frequency ARray}

The LOw Frequency ARray (LOFAR) is a radio antenna array which consists of 50 stations in Northern Europe with a denser core in the Northern Netherlands. Each Dutch station consists of 96 Low Band Antennas (LBAs) and 48 High Band Antennas (HBAs) which operate in the frequency range $10-90 \mathrm{MHz}$ and $110-240 \mathrm{MHz}$, respectively. The 24 stations which form the LOFAR core are located in the Northern Netherlands, and cover a circle of approximately $2 \mathrm{~km}$ radius. In the central area, six stations, also called Superterp, are located in a circular area of roughly $320 \mathrm{~m}$ diameter, and form the densest area of antennas. The layout of LOFAR central stations is shown in figure 1, together with a picture of one LBA. The LOFAR central array is also instrumented with 20 scintillator detectors, the LORA array [12]. Triggers for cosmic ray data acquisition are provided by LORA, which also permits the reconstruction of the arrival direction and energy of primary particles. In the current configuration the LOFAR array allows for the detection of cosmic rays in the energy range $10^{16}-10^{18} \mathrm{eV}$.

Measurements of cosmic rays are performed mostly by using signals from LBAs. The LBAs are designed to operate between $10 \mathrm{MHz}$, where the ionospheric cut-off of radio wavelengths takes place, and $90 \mathrm{MHz}$ where the commercial FM radio band starts. Nevertheless, due to the presence of strong Radio Frequency Interference (RFI) at the lowest frequencies, and the proximity of the FM band at the highest ones, the LBA operational range is limited between $30-80 \mathrm{MHz}$. Each LBA consists of two dipole arms oriented in the NE-SW and NW-SE direction. 

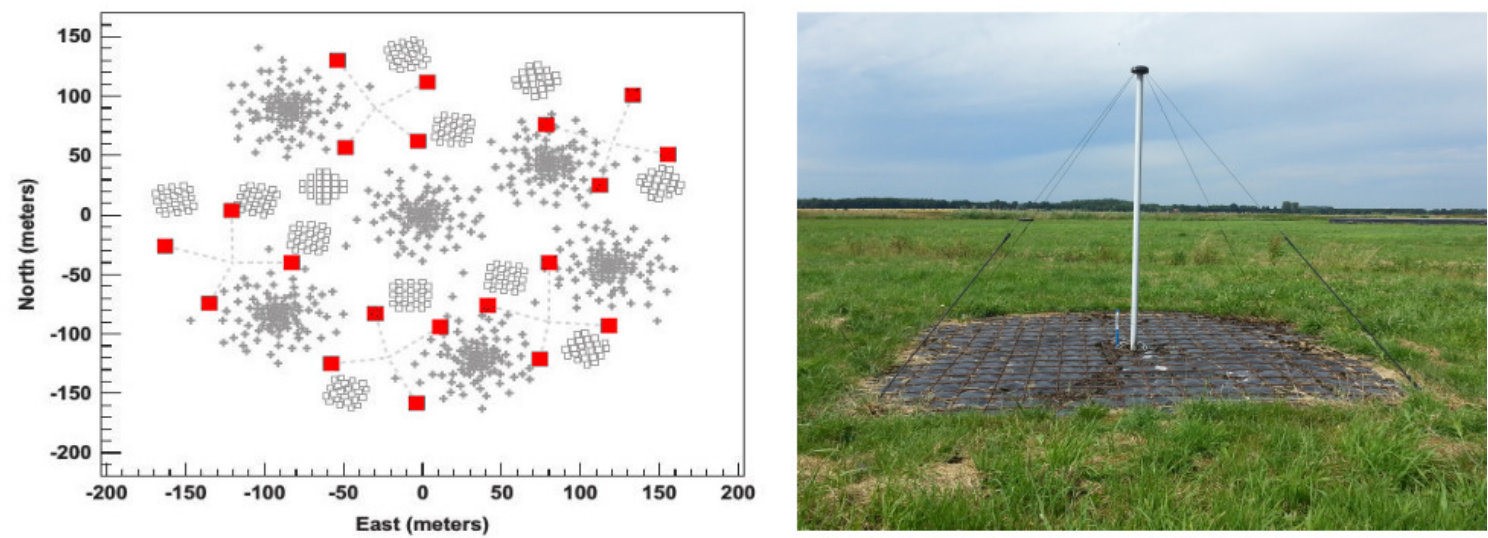

Figure 1: Left: layout of the six LOFAR central stations, the so-called Superterp. The location of LBA inner and outer antenna set is depicted as grey crosses; the position of the HBAs is shown as well (open grey squares). The red squares indicate the position of LORA scintillator detectors. Right: picture of one LBA.

\section{Radio emission processes of extensive air showers}

Secondary charged particles, produced in the atmosphere by the interaction of primary cosmic rays with the atmospheric nuclei, emit radio signals. Radio emission is generated by two mechanisms, the geomagnetic and the charge excess process. In the geomagnetic process, secondary electrons and positrons in the cascade are accelerated in opposite directions due to the Earth magnetic field. This effect creates a current which is linearly polarized in the direction perpendicular to the shower axis and to the geomagnetic field. In the charge excess process (also called Askaryan effect [13]), the radio emission is instead produced by a negative charge excess created at the shower front. In this latter case, the negative charge excess is caused by electrons which are knocked-out and start moving with the cascade, and by the annihilation of secondary positrons with electrons. The radiation emitted due to the charge excess process is polarized in the radial direction with respect to the shower axis. Thus, the combination of these two mechanisms creates an asymmetric distribution of the total radio signal around the shower axis $[14,15]$.

\section{Analysis and results}

In order to study a correlation between the radio frequency spectrum and EAS characteristics, the radio signal in the frequency-domain has been investigated on events detected by LOFAR since 2011. The analysis method used is the following:

- the signal intensity in the time-domain is converted into the frequency-domain by applying a Fast Fourier Transform (FFT). The FFT is calculated on a time-window of 128 samples $(1 \mathrm{sample}=5 \mathrm{~ns})$ corresponding to $640 \mathrm{~ns}$ time-window defined as $\left[\mathrm{t}_{0}-240 \mathrm{~ns}, \mathrm{t}_{0}+400 \mathrm{~ns}\right]$ around the signal peak; the FFT squared module is then calculated (hereafter, $|\mathrm{FFT}|^{2}$ signal $)$

- the background contribution is calculated on a longer time-window of $2^{16}$ samples, equal to $328 \mu \mathrm{s}$, centred on the pulse peak; this long time-window is divided in about 400 sub- 

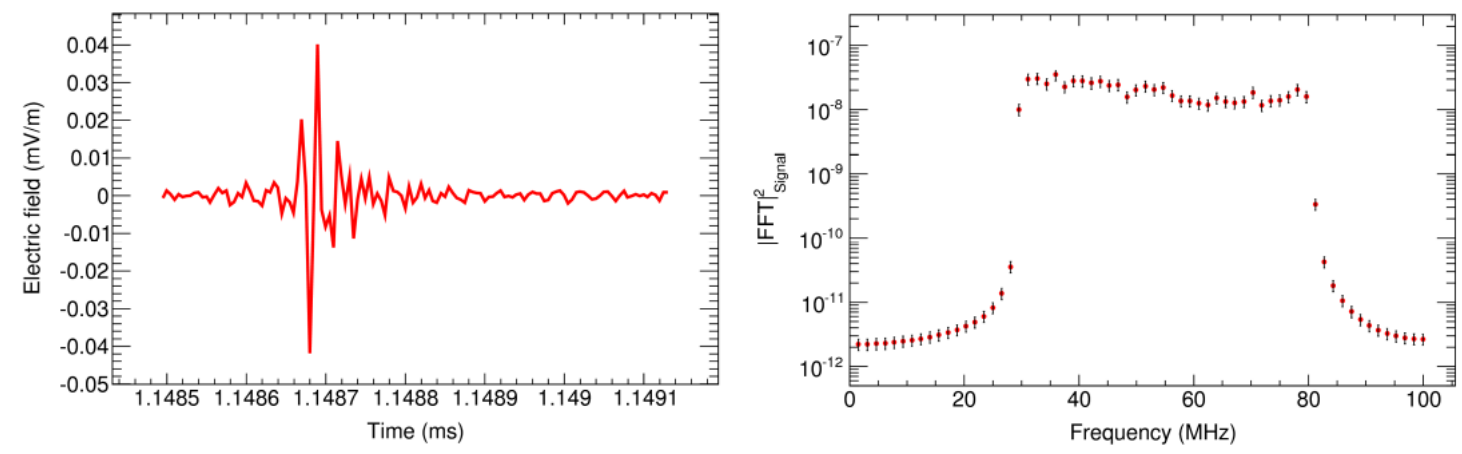

Figure 2: Left: electric field in the time-domain as measured by one LBA in the Superterp. The maximum intensity has a time length of about $50 \mathrm{~ns}$, while the entire window is $640 \mathrm{~ns}$ long, and it is the one used in this analysis. Right: corresponding signal in the frequency-domain; the error bars on the $|\mathrm{FFT}|^{2}$ correspond to an uncertainty of $20 \%$ which has been evaluated through a calibration analysis.

windows of 128 samples, after having excluded the pulse region. The $|F F T|^{2}$ background is then calculated by averaging the $|\mathrm{FFT}|^{2}$ on each of these sub-windows;

- the final $|\mathrm{FFT}|^{2}$ is evaluated as $|\mathrm{FFT}|^{2}=|\mathrm{FFT}|^{2}$ signal $-|\mathrm{FFT}|^{2}{ }_{\text {background }}$

Following the above method, the frequency spectrum has been characterised by fitting the $\log _{10}|\mathrm{FFT}|^{2}$ with a linear function and looking for correlations between the slope of the linear fit and certain parameters. Figure 2 shows, for one event, the electric field measured by one antenna in the time-domain (left), and the corresponding signal in the frequency-domain (right) where the antenna band-filters at $30 \mathrm{MHz}$ and $80 \mathrm{MHz}$ are clearly visible. Errors on the $|\mathrm{FFT}|^{2}$ have been evaluated from data taken during a calibration campaign performed in May 2014. The calibration measurements have been performed using an external emitting source positioned over one inner antenna of one Dutch station, and a difference of $20 \%$ in the receiving power between the two dipoles has been found. This uncertainty has been used as an error on the evaluation of the $|\mathrm{FFT}|^{2}$ in this analysis (see figure 2-right). Figure 3-left shows the distribution of $\log _{10}|\mathrm{FFT}|^{2}$ as function of frequency for one antenna of one event after the background subtraction. Due to the presence of many RFI around $30 \mathrm{MHz}$, which are removed during the data reduction process, most of the antennas displays a low radio signal intensity at $30 \mathrm{MHz}$. For this reason, the first point of the linear-fit region has been considered at $33 \mathrm{MHz}$. Furthermore, during this study, it has been observed that the frequency spectra measured in all antennas increase above $70 \mathrm{MHz}$. This is expected to be caused by the low-pass filter in the different antennas. Therefore, in order to not introduce any bias in the fitting procedure, the last point of the linear-fit region has been chosen at $70 \mathrm{MHz}$.

For each event, the linear-fit has been applied to all the antennas, in order to study the dependence of the slope parameter as function of distance to the shower axis. The antenna position at ground has been projected onto the plane perpendicular to the particle arrival direction vector $\vec{v}$ (hereafter, shower plane). Since the linear-fit method is very sensitive to the signal-to-noise ratio detected in one shower event, strict selection criteria have been used in order to select good events for this analysis. Events have been selected by requiring that, at least in one station, half of the antennas show a pulse-peak value larger than $10 \sigma$, where $\sigma$ is computed for each antenna in 

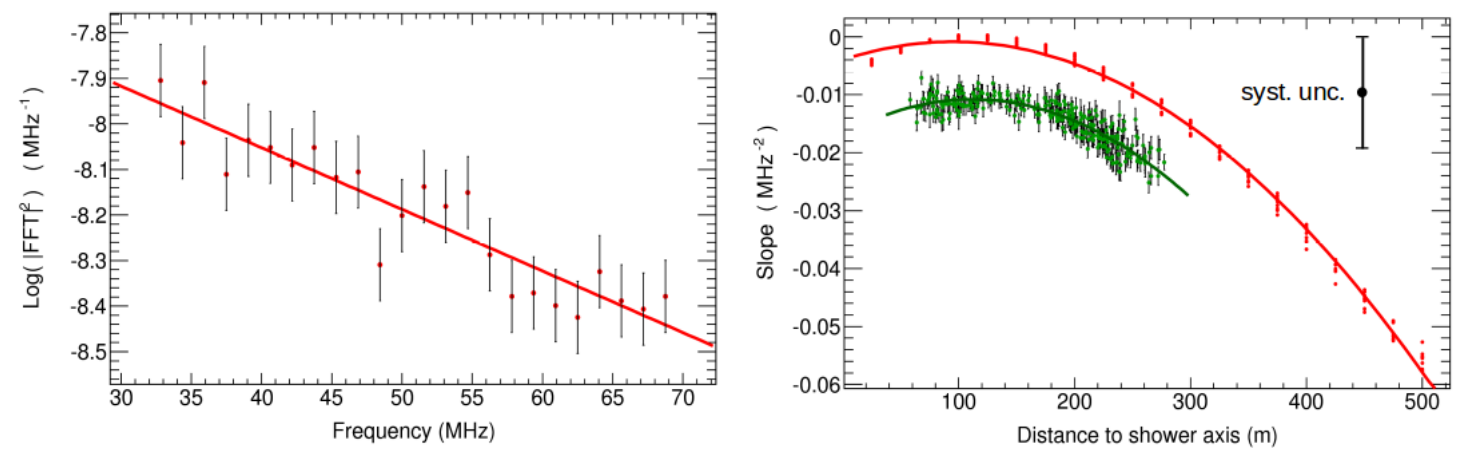

Figure 3: Left: distribution of $\log _{10}|\mathrm{FFT}|^{2}$ as function of frequency for one antenna of one detected event; the distribution has been fitted with a linear function, taking into account the $20 \%$ uncertainty on the $|\mathrm{FFT}|^{2}$ converted into logarithmic values. Right: distribution of the linear-fit slope as function of distance to the shower axis in the shower plane for one event (green points). The uncertainties on the slope values are obtained from the previous linear-fit procedure; the systematic uncertainties from the calibration procedures are shown on the top-right corner. Comparison with the corresponding simulated event is also shown (red points). Both distributions have been fitted with a parabolic function (solid line).

the long time-window of $328 \mu \mathrm{s}$, as described previously. To these pre-selected events, for every frequency $v$ in the range $33-70 \mathrm{MHz}$, the following criterion has been applied:

$$
\left|F F T\left(v_{i}\right)\right|^{2} \text { Signal }-\left|F F T\left(v_{i}\right)\right|^{2} \text { Background }>R M S\left(\left|F F T\left(v_{i}\right)\right|^{2} \text { Background }\right)
$$

Events with less than 10 antennas satisfying condition 4.1 have been discarded. By using these selection criteria, 103 events have been selected and used for the analysis presented here. All the 103 selected events have been compared with the corresponding simulated events. CORSIKA simulations have been produced by using, as input, proton as primary particle, and energy and arrival direction as reconstructed from the real event. The antenna layout used in simulations (see figure 4) has been chosen in order to have a symmetric star-shape around the shower axis on the shower plane. Each simulated event has 160 antennas displayed at $25 \mathrm{~m}$ distance between each others on the shower plane, and distributed on 8 arms, thus covering an area of $500 \mathrm{~m}$ radius around the shower axis.

Figure 3-right shows the linear-fit slope as function of distance to the shower axis for one selected event (green points) together with one simulated event having the value of $\mathrm{X}_{\max }$ closest to the observed one (red points). This event has a primary energy of $(1.7 \pm 0.8) \cdot 10^{17} \mathrm{eV}$ as reconstructed by the LORA scintillator array, and a value of the atmospheric depth where the cascade reaches its maximum development $X_{\max }=(757 \pm 38) \mathrm{g} / \mathrm{cm}^{2}$ as reconstructed by the radio Lateral Distribution Function (LDF) method [16]. The simulated event has $X_{\max }=755 \mathrm{~g} / \mathrm{cm}^{2}$.

As visible in figure 3-right, the distribution of the slope parameter as function of distance to the shower axis is well described by a parabolic function for both real data and simulations. The displayed error bars on the slope parameters have been extrapolated from the fitting procedure. Moreover, systematic uncertainties on the slope values (about $0.01 \mathrm{MHz}^{-2}$ ) have been evaluated from the two antenna calibration procedures used at LOFAR [17], and are indicated on the top-right corner of the figure. Both distributions display a maximum at a distance around $100 \mathrm{~m}$, in agree- 

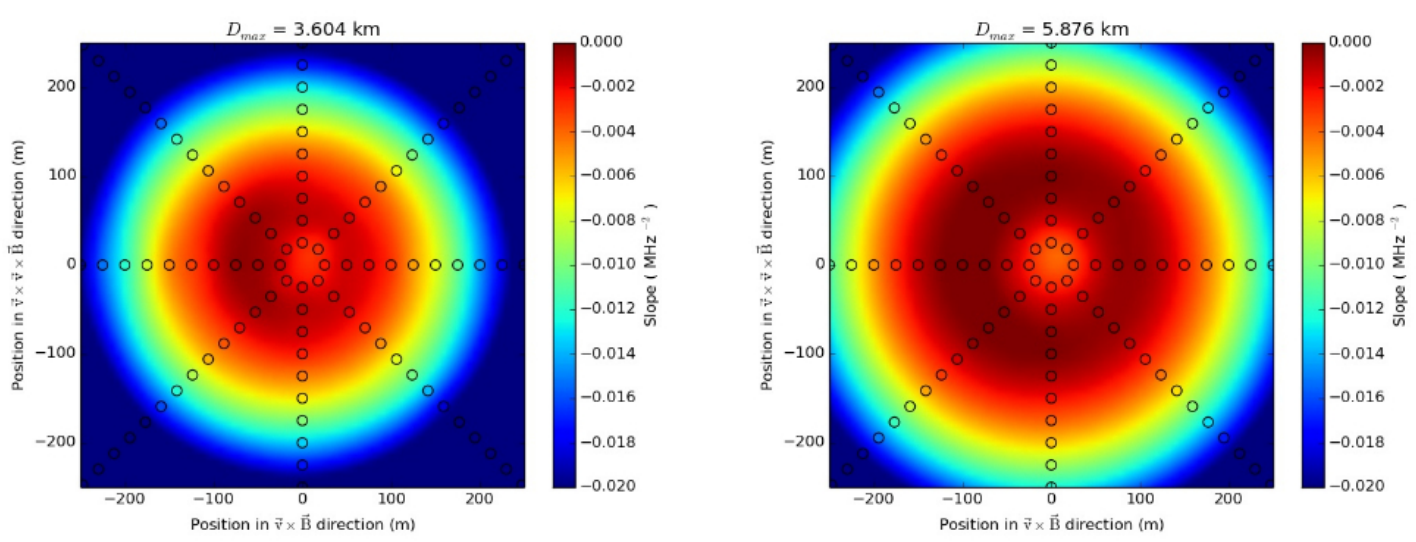

Figure 4: Distribution of the linear-fit slope on the shower plane for two simulated events with $\mathrm{D}_{\max }=3.6 \mathrm{~km}$ (left) and $\mathrm{D}_{\max }=5.9 \mathrm{~km}$ (right). In both figures values of the 160 antennas (open circles) have been interpolated in order to create a smooth background.

ment with the expectations from the radio Cherenkov ring, i.e. the region where the coherence of the radio signal is the uppermost. Nevertheless, the slope distribution for data shows a shift towards smaller slope values. Investigation of this shift is still on-going.

The analysis performed shows also that the slope parameter depends on the geometrical distance of the observer from $\mathrm{X}_{\max }$ (hereafter $D_{\max }$ ). Figure 4 shows the distribution of the slope parameter on the shower plane for two simulated events having a value of $\mathrm{D}_{\max }=3.6 \mathrm{~km}(\mathrm{left})$ and $\mathrm{D}_{\max }=5.9 \mathrm{~km}$ (right) respectively. For all the 103 selected events, the linear-fit procedure has been applied, and only events with a good parabolic fit of the slope parameter as function of distance to the shower axis have been considered. Figure 5 shows the distribution of the slope parameter as function of $\mathrm{D}_{\max }$ at $180 \mathrm{~m}$ and $220 \mathrm{~m}$ distance from the shower axis (blue points). The distributions have been fitted with the following function:

$$
\operatorname{slope}\left(D_{\max }\right)=\frac{\alpha}{1+\exp \left(-\beta \cdot D_{\max }\right)}-\gamma
$$

This fit-function has been previously used on AERA data as described in [11], and has been adjusted to LOFAR data by adding a third free parameter. This was needed because of the different geographical location and different antenna design. Distributions shown in figure 5 display a good agreement between the data points and the fit-function, even if the reliability of the fit is affected by the large uncertainties on $\mathrm{D}_{\max }$ (about $300 \mathrm{~m}$ ). A full parametrization of the frequency spectrum as function of $D_{\max }$ is still under investigation.

\section{Conclusions and outlook}

The radio frequency spectrum in the $30-70 \mathrm{MHz}$ range has been studied. The analysis has been conducted on cosmic ray radio signals detected by LOFAR and on the corresponding simulated events. Results show a clear dependence of the frequency spectrum on the distance to the 

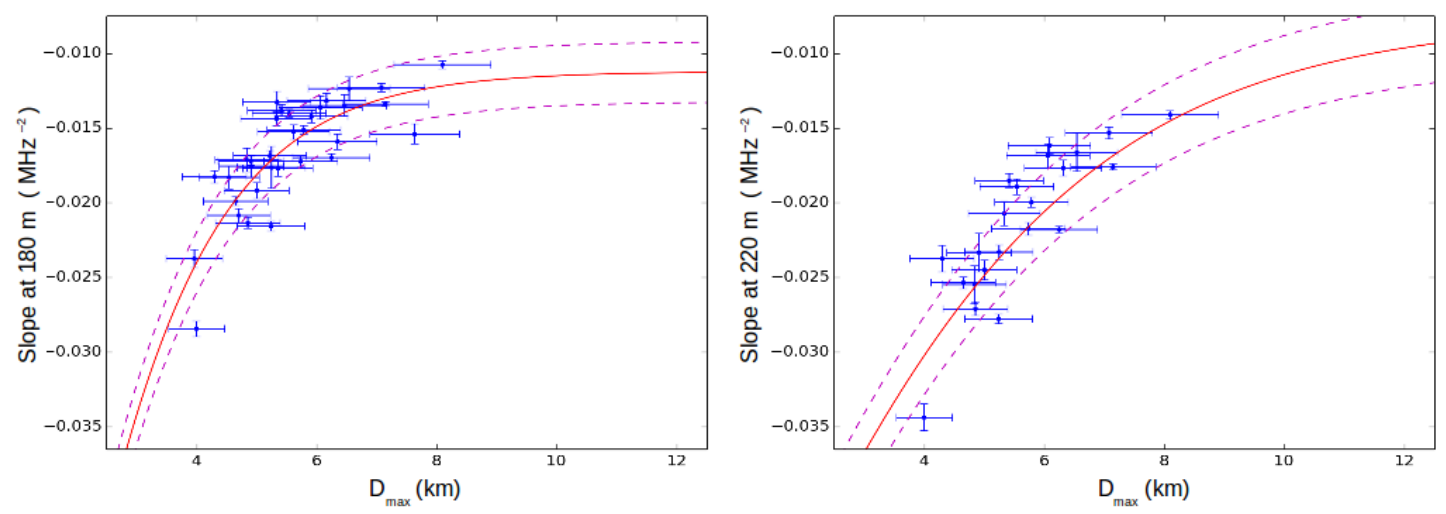

Figure 5: Distribution of the linear-fit slope parameter as function of $\mathrm{D}_{\max }$ at $180 \mathrm{~m}$ (left) and $220 \mathrm{~m}$ (right). Uncertainties on the slope parameter have been extrapolated from the linear-fit procedure. Uncertainties on $\mathrm{D}_{\max }$ have been evaluated from $\sigma_{X_{\max }}=38 \mathrm{~g} / \mathrm{cm}^{2}$ [16]. The red lines indicate the result of the fit obtained using equation 4.2 , with the $68 \%$ confidence region delimited by the dashed purple lines.

shower axis, as predicted by theoretical and analytical calculations. However, the obtained spectral indices for real data are systematically steeper than for simulated showers. This discrepancy is currently under investigation. These characteristics of radio signals can be used as an additional independent method to reconstruct the position of the shower axis at ground. Studies about this option are on-going [18].

Furthermore, a dependence of the frequency spectrum on the geometrical distance to the shower maximum $\mathrm{D}_{\max }$ has been investigated. Results show that, at a fix distance from the shower axis, the slope parameter of the frequency spectrum increases as $D_{\max }$ increases. This is related to the fact that the majority of the radio emission is emitted at an height around $\mathrm{X}_{\max }$, and then propagates with an opening angle of $\sim 1^{\circ}$ given by the atmosphere refractive index. Thus, if the primary interaction happens at higher altitude, the radio footprint is projected from a higher altitude on a larger surface. A study on how to use the frequency spectrum as an independent method to reconstruct $\mathrm{X}_{\max }$ for events detected by LOFAR is still on-going.

\section{Acknowledgments}

The LOFAR cosmic ray key science project acknowledges funding from an Advanced Grant of the European Research Council (FP/2007-2013) / ERC Grant Agreement n. 227610. The project has also received funding from the European Research Council (ERC) under the European Union's Horizon 2020 research and innovation programme (grant agreement No 640130). We furthermore acknowledge financial support from FOM, (FOM-project 12PR3041-3) and NWO (Top Grant 614001-454, and Spinoza Prize SPI 78-409). AN is supported by the DFG (research fellowship NE 2031/1-1).

LOFAR, the Low Frequency Array designed and constructed by ASTRON, has facilities in several countries, that are owned by various parties (each with their own funding sources), and that 
are collectively operated by the International LOFAR Telescope foundation under a joint scientific policy.

\section{References}

[1] J.V. Jelley et al., Radio pulses from extensive cosmic-ray air showers, Nature 205 (1965) 327-328

[2] D. Ardouin et al., Radio-detection signature of high-energy cosmic rays by the CODALEMA experiment, Nucl. Instr. Meth. Phys. Res., A 555 (2005) 148-163 [astro-ph/ 0504297 ]

[3] H. Falcke et al. Detection and imaging of atmospheric radio flashes from cosmic ray air showers, Nature 435 (2005) 313-316

[4] M.P. van Haarlem et al., LOFAR: The Low-Frequency ARray, A\&A 556 A2 (2013) 53 [astro-ph.IM/1305.3550]

[5] A. Aab et al., The Pierre Auger Cosmic Ray Observatory, Nucl. Instr. Meth. Phys. Res., A 798 (2015) 172-213 [astro-ph. IM/1502.01323]

[6] P.A. Bezyazeekov et al. Measurement of cosmic-ray air showers with the Tunka Radio Extension (Tunka-Rex), NIM, A 802 (2015) 89-96 [astro-ph. IM/1509. 08624]

[7] H.R. Allan, Radio Emission From Extensive Air Showers, Prog. in Element. Part. and Cos. Ray Phys. 10 (1971) 171-302

[8] H. Falcke and P.W. Gorham, Detecting Radio Emission from Cosmic Ray Air Showers and Neutrinos with a Digital Radio Telescope, Astroparticle Physics 19 (2003) 477-494

[9] T. Huege and H. Falcke, Radio-Emission from Cosmic Ray Air Showers: Coherent Geosynchrotron Radiation, A\&A 412 (2003) 19-34

[10] T. Huege and H. Falcke, Radio emission from cosmic ray air showers. Monte Carlo simulations, A\&A 430 (2005) 779-798

[11] S. Jansen, PhD thesis, Radio for the Masses - Cosmic ray mass composition measurements in the radio frequency domain (2016) ISBN 9789402800739

[12] S. Thoudam et al., LORA: A scintillator array for LOFAR to measure extensive air shower, Nucl. Instr. Meth. Phys. Res. A 767 (2014) 339 [physics.ins-det/1408.4469]

[13] G.A. Askaryan, Excess Negative Charge of an Electron-Photon Shower and its Coherent Radio Emission, JETP, 14 (1962) 441-443

[14] O. Scholten, K. Werner, and F. Rusydi, A macroscopic description of coherent geo-magnetic radiation from cosmic-ray air showers, Astroparticle Physics 29: 94-103, 2008 [ast ro-ph/0709.2872]

[15] K. Werner and O. Scholten, Macroscopic Treatment of Radio Emission from Cosmic Ray Air Showers based on Shower Simulations, Astroparticle Physics 29 (2008) 393-411 [astro-ph/ 0712 . 2517]

[16] A. Nelles et al., The radio emission pattern of air showers as measured with LOFAR - a tool for the reconstruction of energy and shower maximum, JCAP 05 (2015) 18 [astro-ph. HE/1411. 7868]

[17] A. Nelles et al., Calibrating the absolute amplitude scale for air showers measured at LOFAR, JINST 10 (2015) P11005 [astro-ph. IM/1507. 08932]

[18] A. Bonardi et al., Study of the LOFAR self-trigger and single-station acquisition mode, in proceedings of $35^{\text {th }}$ ICRC, POS (ICRC2017) 402 (2017) 